\title{
Two matrix methods for solution of nonlinear and linear Lane-Emden type equations with mixed condition by operational matrix
}

\author{
Behrooz Basirat*, Mohammad Amin Shahdadi \\ Department of Mathematics, Birjand Branch, Islamic Azad University, Birjand, Iran \\ *Corresponding author E-mail: behrooz.basirat@iaubir.ac.ir
}

\begin{abstract}
Copyright (c)2015 Behrooz Basirat and Mohammad Amin Shahdadi. This is an open access article distributed under the Creative Commons Attribution License, which permits unrestricted use, distribution, and reproduction in any medium, provided the original work is properly cited.
\end{abstract}

\begin{abstract}
The aim of this article is to present an efficient numerical procedure for solving Lane-Emden type equations. We present two practical matrix method for solving Lane-Emden type equations with mixed conditions by Bernstein polynomials operational matrices (BPOMs) on interval $[a, b]$. This methods transforms Lane-Emden type equations and the given conditions into matrix equation which corresponds to a system of linear algebraic equations. We also give some numerical examples to demonstrate the efficiency and validity of the operational matrices for solving Lane-Emden type equations(LEEs).
\end{abstract}

Keywords: Bernstein polynomials, Lane-Emden type equation, Operational matrices.

\section{Introduction}

Lane-Emden type equation is an ordinary differential equation which describes the equilibrium density distribution in self-gravitating sphere of polytrophic isothermal gas and has a singularity at the origin. Many real life phenomena in mathematical physics and astrophysics can be modeled by Lane-Emden type equations such as thermal explosions [1], stellar structure [2], and the thermal behavior of a spherical cloud of gas, isothermal gas spheres and thermionic currents [3].

In recent years, Many powerful methods have been presented for solving of Lane-Emden type equations. For instance, the homotopy perturbation method $[4,6]$, the Legendre wavelets [7], the variational iteration method $[8,9]$, the B-spline method [10], the Adomian decomposition method [11], the Bessel collocation method [12], the Pade series method [13], the rational Legendre pseudospectral method [14], the nonperturbative approximate method [15], the Hermite functions collocation method [16], and the variational approach method [17].

Continuous or piecewise polynomials are incredibly useful mathematical tools as they are precisely defined, calculated rapidly on a modern computer system and can represent a great variety of functions. They can be differentiated and integrated without difficulty. The aim of the present paper is to apply Bernstein operational matrices to propose a reliable numerical technique for solving linear and nonlinear Lane-Emden equations. Some special cases of the problem are solved to show its validity and efficiency in comparison with other existing numerical methods. The approximate solution obtained by the proposed method shows its superiority on the other existing numerical 
solution.

In this work we used that operational matrices for numerical solution of nonlinear Lane-Emden equation,

$u^{\prime \prime}(x)+\frac{\alpha}{x} u^{\prime}(x)+f(x, u)=g(x), \quad \alpha, x \geq 0$,

under the mixed conditions

$\sum_{k=0}^{1}\left(a_{i k} u^{(k)}(a)+b_{i k} u^{(k)}(b)\right)=\lambda_{i}, \quad i=0,1$,

where $a_{i k}, b_{i k}, \lambda_{i}$ and $\alpha$ are constant. $f(x, u)$ is a nonlinear function of $x$ and $u(x)$, whereas $u(x)$ is unknown. The aim of this study is to find the solution of the problem as the truncated Bernstein series defined by

$u(x)=\sum_{n=0}^{N} u_{n} B_{n, N}(x), \quad a \leq x \leq b$,

where $B_{n, N}(x), \quad n=0,1,2, \ldots, N$ denotes the Bernstein polynomials; $u_{n}, \quad 0 \leq n \leq N$ are unknown Bernstein coefficients, and $N$ is any positive integer chosen such that $N \geq 2$. To find a numeric solution in the form (3) of the problem (1), we use the collocation points defined by

$x_{i}=a+\frac{b-a}{2 N-1} i, \quad i=0,1, \ldots, N$.

The Bernstein basis polynomials of degree $N$ are defined by:

$B_{n, N}(x)=\left(\begin{array}{c}N \\ n\end{array}\right) \frac{(x-a)^{n}(b-x)^{N-n}}{(b-a)^{N}}, \quad a \leq x \leq b, \quad n=0,1, \ldots, N$.

The Bernstein polynomials $B_{n, N}(x)$ in the matrix form as follows

$\Phi(x)=\left[B_{0, N}(x), B_{1, N}(x), \ldots, B_{N, N}(x)\right]$.

This paper is organized as follows: first, we introduce Lane-Emden type equations and Bernstein polynomials. In Section 2 we apply Bernstein polynomials approximation and their operational matrices together with collocation method to reduce the Lane-Emden type equation to a system of nonlinear or linear equations. Section 3 exhibits the final system. Section 4 we present a special case. Section 5 illustrates some numerical examples to show the accuracy of this method. Finally Section 6 concludes the paper.

\section{Fundamental relations}

\subsection{Function approximation}

\subsubsection{Approximate of $u(x)$}

A function $u(x)$, square integrable in $[a, b]$, may be expressed in terms of Bernstein basis [18]. In practice, only the first $(N+1)$-terms Bernstein polynomials are considered. Hence, if we write

$u(x) \simeq \sum_{i=0}^{N} u_{i} B_{i, N}(x)=\Phi(x) U$,

where

$U=\left[u_{0}, u_{1}, \ldots, u_{N}\right]^{T}$,

then

$U=\mathbf{Q}^{-1}(\Phi(x), u(x))$, 
where $\mathbf{Q}$ is said dual matrix of $\Phi(x)$ and is given in [18]. Now, we can write the unknown function $u(x)$ in the matrix form as follows

$u(x)=\Phi(x) U$,

Using the expression (5) and taking $n=0,1, \ldots, N$, we find the corresponding matrix relation as

$\Phi(x)=\Delta(x) A$,

where

$\Delta(x)=\left[1, x, \ldots, x^{N}\right]$,

and $A$ is the $(N+1) \times(N+1)$ matrix. For example, on the interval $[0,1]$ and $[-1,1]$, we have

$$
\begin{aligned}
& A_{[0,1]}=\left[\begin{array}{ccccc}
(-1)^{0}\left(\begin{array}{c}
N \\
0
\end{array}\right) & 0 & & \ldots & 0 \\
\vdots & \ddots & & \\
(-1)^{1}\left(\begin{array}{c}
N \\
0
\end{array}\right)\left(\begin{array}{c}
N-0 \\
1
\end{array}\right) & & (-1)^{0}\left(\begin{array}{c}
N \\
n
\end{array}\right) & & \vdots \\
\vdots & & \ddots & & 0 \\
(-1)^{N-0}\left(\begin{array}{c}
N \\
0
\end{array}\right)\left(\begin{array}{c}
N-0 \\
N-0
\end{array}\right) & \ldots & (-1)^{N-n}\left(\begin{array}{c}
N \\
n
\end{array}\right)\left(\begin{array}{c}
N-n \\
N-n
\end{array}\right) & \ldots & (-1)^{0}\left(\begin{array}{c}
N \\
N
\end{array}\right)
\end{array}\right] \text {, } \\
& A_{[-1,1]}=\left[\begin{array}{cccc}
(-1)^{0}\left(\begin{array}{c}
N \\
0
\end{array}\right) & (-1)^{0}\left(\begin{array}{c}
N-1 \\
0
\end{array}\right) & \ldots & \left(\begin{array}{c}
N \\
0
\end{array}\right) \\
(-1)^{1}\left(\begin{array}{c}
N \\
1
\end{array}\right) & (-1)^{1}\left(\begin{array}{c}
N-1 \\
1
\end{array}\right)+(-1)^{0}\left(\begin{array}{c}
N-1 \\
0
\end{array}\right) & \ldots & \left(\begin{array}{c}
N \\
1
\end{array}\right) \\
\vdots & \vdots & \ddots & \vdots \\
(-1)^{N}\left(\begin{array}{c}
N \\
N
\end{array}\right) & (-1)^{N-1}\left(\begin{array}{c}
N-1 \\
N-1
\end{array}\right) & \ldots & \left(\begin{array}{c}
N \\
N
\end{array}\right)
\end{array}\right] .
\end{aligned}
$$

From relations (10), (6) and (11), we obtain the following matrix forms

$u(x)=\Phi(x) U=\Delta(x) A U$.

\subsubsection{Approximate of $f(x, u)$}

We can also approximate the function $f(x, u)$ by the Bernstein polynomials as

$f(x, u) \simeq f(x, \Phi(x) U)=\Phi(x) H$,

where the unknown is

$H=\left[h_{0}, h_{1}, \ldots, h_{N}\right]^{T}$,

similarly (9), we have

$H=\mathbf{Q}^{-1}(\Phi(x), f(x, \Phi(x) U))$.

\subsection{Matrix relations for higher order derivatives of $u(x)$ based on $\Delta(x)$}

We consider the solution $u(x)$ and its $k$ th derivative $u^{(k)}(x)$ defined by the truncated Bernstein series (3). Then we can write its $k$ th derivative in the matrix form

$u^{(k)}(x)=\Phi^{(k)}(x) U, \quad k=1,2, \ldots$ 
and

$u^{(k)}(x)=\Delta^{(k)}(x) A U$,

To obtain the matrix $\Delta^{(k)}(x)$ in terms of the matrix $\Delta(x)$, we can use the following procedure:

$\Delta^{(1)}(x)=\Delta(x) M^{1}$,

$\Delta^{(2)}(x)=\Delta^{(1)}(x) M=\Delta(x) M^{2}$,

$\Delta^{(k)}(x)=\Delta^{(k-1)}(x) M=\Delta(x) M^{k}$,

where

$M=\left[\begin{array}{ccccc}0 & 1 & 0 & \ldots & 0 \\ 0 & 0 & 2 & \ldots & 0 \\ \vdots & \vdots & \ddots & \ddots & \vdots \\ 0 & 0 & 0 & 0 & N \\ 0 & 0 & 0 & \ldots & 0\end{array}\right]$

Consequently, by substituting the matrix form (18) and (19) into (17), we have the matrix relation

$u^{(k)}(x)=\Delta(x) M^{k} A U$.

\subsection{Matrix relations for higher order derivatives of $u(x)$ based on $\Phi(x)$}

The differentiation of vector $\Phi(x)$ in Eq. (6) can be expressed as

$\Phi^{\prime}(x)=\Phi(x) \mathbf{D}$,

where $\mathbf{D}$ is the $(N+1) \times(N+1)$ operational matrix of derivatives for Bernstein polynomials. From (11), we have $\Phi(x)=\Delta(x) A$ and then

$\Phi^{\prime}(x)=\left[0,1,2 x, \ldots, N x^{N-1}\right] A$,

Defining $(N) \times(N+1)$ matrix $V$ and vector $\Delta^{*}(x)$ as

$V=\left[\begin{array}{ccccc}0 & 1 & 0 & \cdots & 0 \\ 0 & 0 & 2 & \cdots & 0 \\ 0 & 0 & 0 & \cdots & 0 \\ \vdots & \vdots & \vdots & \ddots & \vdots \\ 0 & 0 & & \cdots & N\end{array}\right], \quad \Delta^{*}(x)=\left[1, x, x^{2}, \ldots, x^{N-1}\right]$,

equation (23) may then be restated as

$\Phi^{\prime}(x)=\Delta^{*}(x) V A$.

We now expand vector $\Delta^{*}(x)$ in terms of $\Phi(x)$. We get $\Delta^{*}(x)=\Phi(x) B^{*}$ where

$B^{*}=\left[A_{[1]}^{-1}, A_{[2]}^{-1}, A_{[3]}^{-1}, \ldots, A_{[N]}^{-1}\right]$,

where $A_{[k]}^{-1}$ is the $(k+1)$ th row of $A^{-1}$ for $k=1,2, \ldots, N$. so

$\Phi^{\prime}(x)=\Phi(x) B^{*} V A$.

Therefore, we have the operational matrix of derivative as

$\mathbf{D}=B^{*} V A$.

If we approximate $u(x) \simeq \Phi(x) U$, then for $k \geq 2$ ( $k$ is the order of derivatives), we get

$u^{(k)}(x) \simeq \Phi^{(k)}(x) U=\Phi(x) \mathbf{D}^{k} U$. 


\subsection{Matrix relations for the mixed conditions}

We obtain the corresponding matrix forms for the conditions (2) by means of the relations (21) as

$\sum_{k=0}^{1}\left[a_{i k} \Delta(a)+b_{i k} \Delta(b)\right] M^{k} A U=\lambda_{i}, \quad i=0,1$.

Briefly, the matrix form for conditions (2) is

$\mathcal{I}_{i} U=\left[\lambda_{i}\right]$,

where

$$
\begin{aligned}
\mathcal{I}_{i} & =\sum_{k=0}^{1}\left[a_{i k} \Delta(a)+b_{i k} \Delta(b)\right] M^{k} A \\
& =\left[z_{i 0}, z_{i 1}, \ldots, z_{i N}\right] .
\end{aligned}
$$

\section{Methods of solution}

We are now ready to construct the fundamental matrix equation corresponding to Eq. (1).

\subsection{First method}

In this method, we substitute and simplify the matrix relations (21) into Eq. (1), and obtain the fundamental matrix equation

$\Delta(x) M^{2} A U+\frac{\alpha}{x} \Delta(x) M A U+\Delta(x) A H=g(x)$.

By plugging the collocation points $x_{i}$ defined by (4), we get the system of matrix equations

$\Delta\left(x_{i}\right) M^{2} A U+\frac{\alpha}{x_{i}} \Delta\left(x_{i}\right) M A U+\Delta\left(x_{i}\right) A H=g\left(x_{i}\right), \quad i=0,1, \ldots, N$,

or briefly the fundamental matrix equation

$\left\{\Delta M^{2} A+\Gamma \Delta M A\right\} U=G-\Delta A H$.

where

$\Delta=\left[\begin{array}{c}\Delta\left(x_{0}\right) \\ \Delta\left(x_{1}\right) \\ \vdots \\ \Delta\left(x_{N}\right)\end{array}\right]=\left[\begin{array}{ccccc}1 & x_{0} & x_{0}^{2} & \ldots & x_{0}^{N} \\ 1 & x_{1} & x_{1}^{2} & \ldots & x_{1}^{N} \\ \vdots & \vdots & \ddots & \vdots & \vdots \\ 1 & x_{N} & x_{N}^{2} & \ldots & x_{N}^{N}\end{array}\right]$,

and

$\Gamma=\left[\begin{array}{ccccc}\frac{\alpha}{x_{0}} & 0 & 0 & \ldots & 0 \\ 0 & \frac{\alpha}{x_{1}} & 0 & \ldots & 0 \\ \vdots & \vdots & \ddots & \vdots & \vdots \\ 0 & 0 & 0 & \ldots & \frac{\alpha}{x_{N}}\end{array}\right], \quad G=\left[\begin{array}{c}g\left(x_{0}\right) \\ g\left(x_{1}\right) \\ \vdots \\ g\left(x_{N}\right)\end{array}\right]$

Hence, Eq. (35) can be written in the form

$\mathbf{W} U=\mathcal{F} \quad$ or $\quad[\mathbf{W} ; \mathcal{F}], \quad \mathbf{W}=\left[w_{p q}\right], \quad p, q=0,1, \ldots, N$, 
where

$\mathbf{W}=\left[w_{p q}\right]=\Delta M^{2} A+\Gamma \Delta M A, \quad \mathcal{F}=G-\Delta A H$,

Finally, to obtain the solution of Eq. (1) under the conditions (2), we replace the row matrices (32) by the last 2 rows of the matrix (38), and get the new augmented matrix

$[\overline{\mathbf{W}} ; \overline{\mathcal{F}}]=\left[\begin{array}{cccccc}\mathrm{w}_{00} & \mathrm{w}_{01} & \ldots & \mathrm{w}_{0 N} & ; & g\left(x_{0}\right)-\Delta\left(x_{0}\right) A H \\ \mathrm{w}_{10} & \mathrm{w}_{11} & \ldots & \mathrm{w}_{1 N} & ; & g\left(x_{1}\right)-\Delta\left(x_{1}\right) A H \\ \ldots & \ldots & & \ldots & ; & \ldots \\ \mathrm{w}_{N, 0} & \mathrm{w}_{N, 1} & \ldots & \mathrm{w}_{N, N} & ; & g\left(x_{N}\right)-\Delta\left(x_{N}\right) A H \\ & & & & & \\ z_{00} & z_{01} & \ldots & z_{0 N} & ; & \lambda_{0} \\ z_{10} & z_{11} & \ldots & z_{1 N} & ; & \lambda_{1}\end{array}\right]$.

If $\operatorname{rank} \overline{\mathbf{W}}=\operatorname{rank}[\overline{\mathbf{W}} ; \overline{\mathcal{F}}]=N+1$, then we can write $U=(\overline{\mathbf{W}})^{-1} \overline{\mathcal{F}}$; thus, the matrix $U$ (thereby the coefficients $u_{0}$, $\left.u_{1}, \ldots, u_{N}\right)$ is uniquely determined. Also the Eq. (1) under the conditions (2) has a unique solution. This solution is given by truncated Bernstein series (3). On the other hand, when $|\overline{\mathbf{W}}|=0$, if $\operatorname{rank} \overline{\mathbf{W}}=\operatorname{rank}[\overline{\mathbf{W}} ; \overline{\mathcal{F}}]<N+1$, then we may find a particular solution. Otherwise if $\operatorname{rank} \overline{\mathbf{W}} \neq \operatorname{rank}[\overline{\mathbf{W}} ; \overline{\mathcal{F}}]=N+1$, then there is no solution.

\subsection{Second method}

In this method, we substitute and simplify the matrix relations (11) and (29) into Eq. (1), and obtain the fundamental matrix equation

$\Delta(x) A D^{2} U+\frac{\alpha}{x} \Delta(x) A D U+\Delta(x) A H=g(x)$,

By plugging the collocation points $x_{i}$ defined by (4), we get the system of matrix equations

$\Delta\left(x_{i}\right) A D^{2} U+\frac{\alpha}{x_{i}} \Delta\left(x_{i}\right) A D U+\Delta\left(x_{i}\right) A H=g\left(x_{i}\right), \quad i=0,1, \ldots, N$,

or briefly the fundamental matrix equation

$\left\{\Delta A D^{2}+\Gamma \Delta A D\right\} U=G-\Delta A H$.

\section{Special case}

\subsection{Linear Lane-Emden equation}

In Eq. (1), if $f(x, u)=p(x) u(x)$ then we have a linear Lane-Emden equation as

$u^{\prime \prime}(x)+\frac{\alpha}{x} u^{\prime}(x)+p(x) u(x)=g(x), \quad \alpha, x \geq 0$.

Similarly nonlinear Lane-Emden equation, we have the fundamental matrix equation as follows

$\left\{\Delta M^{2} A+\Gamma \Delta M A+P \Delta A\right\} U=G$,

where

$P=\left[\begin{array}{ccccc}p\left(x_{0}\right) & 0 & 0 & \ldots & 0 \\ 0 & p\left(x_{1}\right) & 0 & \ldots & 0 \\ \vdots & \vdots & \ddots & \vdots & \vdots \\ 0 & 0 & 0 & \ldots & p\left(x_{N}\right)\end{array}\right]$ 
Table 1: Approximate and exact solutions for Example 1.

\begin{tabular}{ccccc}
\hline$x$ & $\begin{array}{c}\text { Present method } \\
\text { with } N=2\end{array}$ & $\begin{array}{c}\text { Present method } \\
\text { with } N=4\end{array}$ & $\begin{array}{c}\text { Present method } \\
\text { with } N=6\end{array}$ & Exact solution \\
\hline 0.1 & 2.55744 & 3.27765 & 3.27573 & 3.27562 \\
0.2 & 2.52077 & 3.33314 & 3.33143 & 3.33132 \\
0.3 & 2.53269 & 3.42746 & 3.42576 & 3.42566 \\
0.4 & 2.59322 & 3.56270 & 3.56098 & 3.56086 \\
0.5 & 2.70234 & 3.74213 & 3.74040 & 3.74027 \\
0.6 & 2.86006 & 3.97022 & 3.96838 & 3.96824 \\
0.7 & 3.06637 & 4.25260 & 4.25054 & 4.25039 \\
0.8 & 3.32129 & 4.59613 & 4.59386 & 4.59371 \\
0.9 & 3.62480 & 5.00883 & 5.00690 & 5.00676 \\
1.0 & 3.97691 & 5.49992 & 5.50000 & 5.5 \\
\hline
\end{tabular}

\section{Numerical examples}

To illustrate the effectiveness of the proposed method in the present paper, several examples are presented in this section.

Example 1. Consider the linear Lane-Emden equation [19]

$\left\{\begin{array}{l}u^{\prime \prime}(x)+\frac{2}{x} u^{\prime}(x)-4 u(x)=-2, \\ u(1)=5.5, \quad u^{\prime}(0)=0\end{array}\right.$

where the exact solution is $u(x)=0.5+5 \frac{\sinh (2 x)}{x \sinh (2)}$.

Numerical results for $N=2, N=4$ and $N=6$ in the presented method by Bernstein operational matrices are shown in Table 1.

As it can be observed, we have almost obtained the exact solution for $N=6$ through Bernstein operational matrices. The approximated solution for $N=2, N=4$ and $N=6$ are as follows:

$u_{2}(x)=2.642709-1.095698 x+2.429902 x^{2}$,

$u_{4}(x)=3.260101-0.014183 x+1.906748 x^{2}-0.148239 x^{3}+0.495493 x^{4}$,

$u_{6}(x)=3.257315-0.000020 x+1.838468 x^{2}-0.001711 x^{3}+0.373546 x^{4}-0.010367 x^{5}+0.042769 x^{6}$

Since the exact solution is $u(x)=0.5+5 \frac{\sinh (2 x)}{x \sinh (2)}$, if we write Taylor expansion, we will have:

$u(x)=0.5+5 \frac{\sinh (2 x)}{x \sinh (2)} \simeq 3.257206+1.838137 x^{2}+0.367627 x^{4}+0.035012 x^{6}+O\left(x^{8}\right)$

As it can be observed, as $N$ increases, the approximate solution gets closer to the exact solution.

Example 2. Consider the Linear Lane-Emden equation [19]

$$
\left\{\begin{array}{l}
u^{\prime \prime}(x)+\frac{1}{x} u^{\prime}(x)=\frac{64}{\left(x^{2}-8\right)^{2}(\ln (2)+\ln (5))}, \\
u(1)=0, \quad u^{\prime}(0)=0
\end{array}\right.
$$

where the exact solution is $u(x)=2 \log _{10}\left(\frac{7}{8-x^{2}}\right)$. Numerical results for $N=3, N=5$ and $N=7$ in the presented method by Bernstein operational matrices are shown in Table 2 . 
Table 2: Approximate and exact solutions for Example 2.

\begin{tabular}{ccccc}
\hline$x$ & $\begin{array}{c}\text { Present method } \\
\text { with } N=3\end{array}$ & $\begin{array}{c}\text { Present method } \\
\text { with } N=5\end{array}$ & $\begin{array}{c}\text { Present method } \\
\text { with } N=7\end{array}$ & Exact solution \\
\hline 0.1 & -0.113213 & -0.113751 & -0.114463 & -0.114897 \\
0.2 & -0.109783 & -0.110485 & -0.111196 & -0.111630 \\
0.3 & -0.104065 & -0.105010 & -0.105723 & -0.106157 \\
0.4 & -0.096060 & -0.097288 & -0.098002 & -0.098436 \\
0.5 & -0.085768 & -0.087264 & -0.087976 & -0.088407 \\
0.6 & -0.073188 & -0.074875 & -0.075569 & -0.075991 \\
0.7 & -0.058322 & -0.060045 & -0.060688 & -0.061084 \\
0.8 & -0.041168 & -0.042687 & -0.043221 & -0.043559 \\
0.9 & -0.021728 & -0.022706 & -0.023039 & -0.023262 \\
1.0 & $4.441 \times 10^{-16}$ & $-1.437 \times 10^{-15}$ & $-7.936 \times 10^{-16}$ & 0 \\
\hline
\end{tabular}

As it can be observed, we have almost obtained the exact solution for $N=7$ through Bernstein operational matrices. The approximated solution for $N=3, N=5$ and $N=7$ are as follows:

$u_{3}(x)=-0.114357-6.93889 \times 10^{-18} x+0.114357 x^{2}$

$u_{5}(x)=-0.114835-0.000037 x+0.108589 x^{2}+0.000348 x^{3}+0.006810 x^{4}-0.000876 x^{5}$,

$u_{7}(x)=-0.115549-0.0000005 x+0.108573 x^{2}+0.000011 x^{3}+0.006809 x^{4}-0.000043 x^{5}+0.000384 x^{6}$

$-0.000184 x^{7}$.

Since the exact solution is $u(x)=2 \log _{10}\left(\frac{7}{8-x^{2}}\right)$, if we write Taylor expansion, we will have:

$2 \log _{10}\left(\frac{7}{8-x^{2}}\right) \simeq-0.115984+0.108574 x^{2}+0.006786 x^{4}+0.000565 x^{6}+O\left(x^{8}\right)$

As it can be observed, as $N$ increases, the approximate solution gets closer to the exact solution.

Example 3. Consider the nonlinear Lane-Emden equation

$$
\left\{\begin{array}{l}
u^{\prime \prime}(x)+\frac{2}{x} u^{\prime}(x)+e^{u(x)}=\frac{e^{\sin (x)} x-\sin (x) x+2 \cos (x)}{x}, \\
u(-1)=\sin (-1), \quad u^{\prime}(1)=\cos (1),
\end{array}\right.
$$

where the exact solution is $u(x)=\sin (x)$. Numerical results for $N=4, N=6$ and $N=8$ in the presented method by Bernstein operational matrices are shown in Table 3.

As it can be observed, we have almost obtained the exact solution for $N=8$ through Bernstein operational matrices. The approximated solution for $N=4, N=6$ and $N=8$ are as follows:

$$
\begin{aligned}
u_{4}(x) & =-0.000118+0.998664 x+0.000389 x^{2}-0.158528 x^{3}-0.001607 x^{4} \\
u_{6}(x)= & 0.000005+0.999994 x+0.000026 x^{2}-0.166624 x^{3}-0.000224 x^{4}+0.008044 x^{5}+0.000137 x^{6} \\
u_{8}(x)= & 0.00000001+0.999999 x+0.0000002 x^{2}-0.166667 x^{3}-0.000003 x^{4}+0.0083331 x^{5}+0.000007 x^{6} \\
& -0.000194 x^{7}-0.000004 x^{8}
\end{aligned}
$$

Since the exact solution is $u(x)=\sin (x)$, if we write Taylor expansion, we will have:

$\sin (x) \simeq 1.0 x-0.166667 x^{3}+0.008333 x^{5}-0.000198 x^{7}+O\left(x^{9}\right)$

As it can be observed, as $N$ increases, the approximate solution gets closer to the exact solution. 
Table 3: Approximate and exact solutions for Example 3.

\begin{tabular}{ccccc}
\hline$x$ & $\begin{array}{c}\text { Present method } \\
\text { with } N=4\end{array}$ & $\begin{array}{c}\text { Present method } \\
\text { with } N=6\end{array}$ & $\begin{array}{c}\text { Present method } \\
\text { with } N=8\end{array}$ & Exact solution \\
\hline 0.1 & 0.099594 & 0.099838 & 0.099833 & 0.099833 \\
0.2 & 0.198359 & 0.198674 & 0.198669 & 0.198669 \\
0.3 & 0.295223 & 0.295524 & 0.295520 & 0.295520 \\
0.4 & 0.389223 & 0.389419 & 0.389418 & 0.389418 \\
0.5 & 0.479395 & 0.479419 & 0.479425 & 0.479425 \\
0.6 & 0.564771 & 0.564623 & 0.564642 & 0.564642 \\
0.7 & 0.644377 & 0.644176 & 0.644218 & 0.644218 \\
0.8 & 0.717238 & 0.717285 & 0.717356 & 0.717356 \\
0.9 & 0.782374 & 0.783228 & 0.783328 & 0.783327 \\
1.0 & 0.838801 & 0.841358 & 0.841472 & 0.841471 \\
\hline
\end{tabular}

\section{Conclusion}

In this paper, we have proposed a numerical solution to solve Lane-Emden equation with mixed conditions by Bernstein polynomials operational matrices. We use formula for numerical examples and it is obvious that the numerical solution coincides with the exact solution even with a few Bernstein polynomials used in the approximation. Finally, errors show that the approximation becomes more accurate when $N$ is increased. Therefore, for better results, it is recommended to use a larger $N$.

\section{Acknowledgements}

The authors would like to thank Islamic Azad University of Birjand Branch for financially supporting this research and providing facilities and encouraging this work.

\section{References}

[1] P.L. Chambre, "On the solution of the Poisson-Boltzmann equation with application to the theory of thermal explosions", J. Chem. Phys., No.20, (1952), pp.1795-1797.

[2] S. Chandrasekhar, Introduction to the Study of Stellar Structure, Dover, New York, (1967).

[3] O.U. Richardson, The Emission of Electricity from Hot Bodies, Longman, Green and Co., London, New York, (1921).

[4] A. Yildirim, T. Öziş, "Solutions of singular IVPs of Lane-Emden type by homotopy perturbation method", Phys. Lett. A, No.369, (2007), pp.70-76.

[5] J.I. Ramos, "Series approach to the Lane-Emden equation and comparison with the homotopy perturbation method", Chaos Soliton Fractals, No.38, (2008), pp.400-408.

[6] M.S.H. Chowdhury, I. Hashim, "Solutions of Emden-Fowler equations by homotopy-perturbation method", Nonlinear Anal. RWA, No.10, (2009), pp.104-115.

[7] S.A. Yousefi, "Legendre wavelets method for solving differential equations of Lane-Emden type", Appl. Math. Comput., No.181, (2006), pp.1417-1422.

[8] A. Yildirim, T. Öziş, "Solutions of singular IVPs of Lane-Emden type by the variational iteration method", Nonlinear Anal., No.70, (2009), pp.2480-2484.

[9] M. Dehghan, F. Shakeri, "Approximate solution of a differential equation arising in astrophysics using the variational iteration method", New Astron., No.13, (2008), pp.53-59.

[10] N. Caglar, H. Caglar, "B-spline solution of singular boundary value problems", Appl. Math. Comput., No.182, (2006), pp.1509-1513. 
[11] A.M. Wazwaz, "A new algorithm for solving differential equations of Lane-Emden type", Appl. Math. Comput., No.118, (2001), pp.287-310.

[12] Ş. Yüzbaşı, M. Sezer, "A collocation approach to solve a class of Lane-Emden type equations", J. Adv. Res. Appl. Math., Vol.3, No.2, (2011), pp.58-73.

[13] S.K. Vanani, A. Aminataei, "On the numerical solution of differential equations of Lane-Emden type", Comput. Math. Appl., No.59, (2010), pp.2815-2820.

[14] K. Parand, A. Shahini, M. Dehghan, "Rational Legendre pseudospectral approach for solving nonlinear differential equations of Lane-Emden type", J. Comput. Phys., No.228, (2009), pp.8830-8840.

[15] N.T. Shawagfeh, "Nonperturbative approximate solution for Lane-Emden equation", J. Math. Phys., Vol.34, No.9, (1993), pp.43-64.

[16] K. Parand, M. Dehghan, A.R. Rezaei, S.M. Ghaderi, "An approximation algorithm for the solution of the nonlinear Lane-Emden type equations arising in astrophysics using Hermite functions collocation method", Comput. Phys. Comm., No.181, (2010), pp.1096-1108.

[17] Ji-Huan He, "Variational approach to the Lane-Emden equation", Appl. Math. Comput., No.143, (2003), pp.539-541.

[18] K. Maleknejad, E. Hashemizadeh, B. Basirat, "Computational method based on Bernestein operational matrices for nonlinear Volterra- Fredholm-Hammerstein integral equations", Commun. Nonlinear Sci. Numer. Simul., Vol.17, No.1, (2012), pp.52-61.

[19] Ş. Yüzbaşı, M. Sezer, "An improved Bessel collocation method with a residual error function to solve a class of LaneEmden differential equations", Math. Comput. Modelling, No.57, (2013), pp.1298-1311. 